\title{
Mucinous Cystadenoma Arising From Renal Pelvis
}

\author{
SK Biswas ${ }^{1}$, MM Hossain ${ }^{2}$, A Deb $^{3}$
}

\begin{abstract}
:
Primary mucinous cystic tumors occurring in the renal pelvis or the entire pelvicaliceal system are uncommon. Most are mucinous cystadenoma and less frequently mucinous cystadenocarcinomas. In fact, the publications on these neoplasms are limited to a few cases. Because mucinous cystadenomas of kidney are exceedingly rare, little is known about their natural history, pathogenesis and clinicopathologic features. Herein, we describe a case of mucinous cystadenoma arising from the renal pelvis, which resembled hydronephrosis. The patient presented with a history of intermittent dull pain on the left loin for 6 months. He had no history of removing urinary calculus or long- standing chronic infection in urinary system. The histopathology was unique since the inner surface of the cyst was covered by a mucinous epithelium connected to a urothelium, a transition from non- neoplastic urothelium to mucinous epithelium. This suggests that mucinous cystadenoma of renal pelvis, like its malignant counterpart, may arise in foci of metaplastic mucinous areas.
\end{abstract}

Key words: Mucinous cystadenoma, renal pelvis.

\section{Introduction:}

Tumors of the renal pelvis are uncommon, and the most common neoplasms are urothelial in origin. Mucinous cystic neoplasms of kidney are exceedingly rare primary renal neoplasm, with only 22 cases of mucinous cystadenoma and 5 cases of mucinous cystadenocarcinomas have been reported in English literature ${ }^{1,2}$. Mucinous cystadenocarcinomas of renal pelvis was first described in 1956 by Arcadi et $\mathrm{al}^{3}$. Because knowledge about renal mucinous cystic neoplasms is extremely limited and they have not been recognized in the WHO classification of tumors of the urinary system and male genital organs ${ }^{4}$. Here we reported a case of mucinous cystadenoma of kidney, which may be arising from extensive mucinous metaplasia of renal pelvic urothelium secondary to chronic hydronephrosis in a 62 years old patient.

1. Dr. Swapan Kumar Biswas, MBBS, FCPS (Surgery), MRCSEd, Associate Professor, Department of Surgery, Faridpur Medical College.

2. Dr. Muhammad Mofazzal Hossain, MBBS, FCPS (Surgery) Assistant Professor, Department of Surgery, Faridpur Medical College.

3. Dr. Arjun Deb, MBBS, Assistant Registrar, Department of Surgery, Faridpur Medical College Hospital.

Address of correspondence :

Dr. Swapan Kumar Biswas, FCPS (Surgery), MRCSEd, Associate Professor, Department of Surgery, Faridpur Medical College, Faridpur7800, Bangladesh. Phone: $+88-01712120828$,

E-mail: swapan_kb@yahoo.com

\section{Case report:}

A 62 years old male presented with a history of left sided loin swelling and dull aching pain for the last 6 months. Recently the patient complained of rapid increase in size of the swelling. Ultrasonographic examination of the abdomen revealed enlarged left kidney with gross hydronephrosis with complex mass $(13.9 \mathrm{~cm} \times 11 \mathrm{~cm})$ in left kidney. He had no history of hematuria, dysuria, frequent micturition or fever during the past 6 months. Contrast-enhanced abdominal CT scans showed a large well defined soft tissue mass $(13.3 \times 11.2 \mathrm{~cm})$ in retroperitoneal region not separable from left kidney. Incomplete rim of calcification is noted within the lesion. The lesion is extending up to anterior abdominal wall on left side. Pelvicaliceal system of left kidney is dilated with thinning of renal parenchyma. It did not reveal any other mass lesions or ascites in the abdomen. The IVU test displayed passage of no contrast through the left renal parenchyma, showing almost nonfunctional state. As for laboratory examination, including routine blood test, urine test, renal function tests and other biochemical examination were all within the normal ranges. 


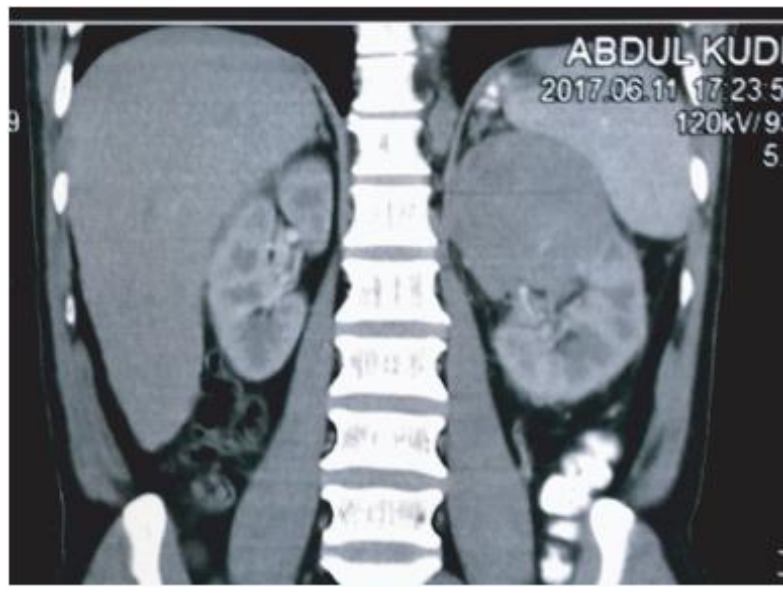

Figure 1: CT scan of abdomen.

With above findings we planned for excision of retroperitoneal mass with left sided nephrectomy. Abdomen was opened by long left paramedian incision. After mobilizing left side colon we found a huge cystic swelling with two protuberances (Fig. 2). The cortex of the kidney was thinned out to a fibrous rim and attached to the cystic swelling. Left renal artery and vein were ligated and the mass was excised. On cut section of the mass, it was cystic involving the renal pelvis and calyces, which was filled with greyish-green gelatinous mucus. The cortex of the kidney was thinned out to a fibrous rim. A diagnosis of mucinous cystadenoma arising from the renal pelvis was made on histopathology report. During the 15 months follow-up, the patient was well and no recurrence of the neoplasm occurred.

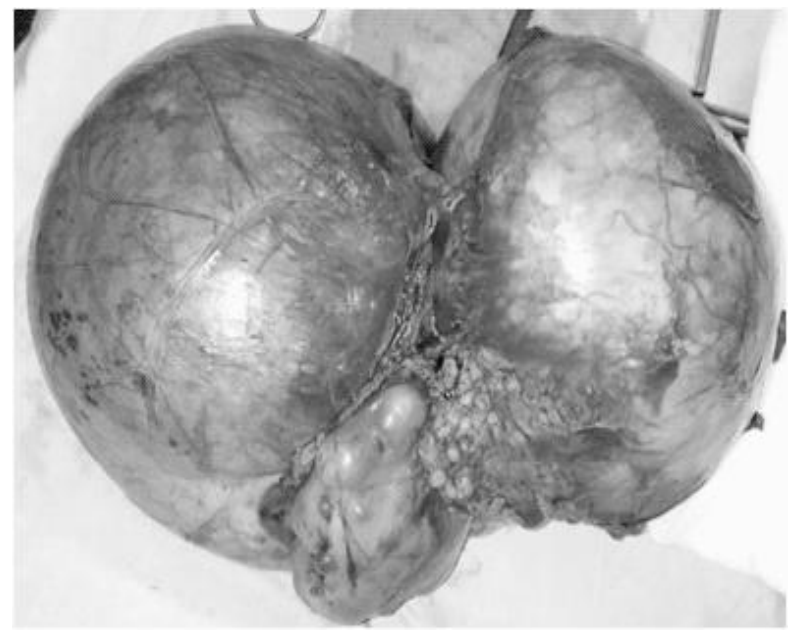

Figure 2: Excised mass

\section{Discussion:}

Primary mucinous epithelial tumor of kidney is an extremely rare neoplasm, only isolated case reports were documented in the English literature. ChabléMontero $\mathrm{F}$ et $\mathrm{al}^{2}$ reported 3 cases of mucinous cystadenoma of the pyelocaliceal system in 2013. They analyzed previously published 17 cases of mucinous cystadenoma arising from renal pelvis in English language. In addition, they also found 5 cases of primary mucinous cystadenocarcinomas of kidney in literature. Mitome $\mathrm{T}$ et $\mathrm{al}^{3}$ reported another case of mucinous cystadenoma in a horseshoe kidney in 2015. Most tumors occurred in native kidney; however, 3 cases of mucinous cystadenoma were occurred in horseshoe kidney ${ }^{2,5,6}$. The neoplasms were unilocular or multilocular with abundant mucin deposits. Some cases showed focal calcifications, granular, nodular or papillary structures attached to the inner surface of the cystically dilated areas. Forty-five percent cases were associated with renal lithiasis ${ }^{1}$ Mucinous cystadenomas occurred mainly in men (male to female ratio was 11:7), ranging in age from 27 to 79 years (median age, 59 years; mean age, 54 years). The mean size of the tumors was $15.6 \mathrm{~cm}$ (range, $2.4-37 \mathrm{~cm}$ ). Some patients had symptoms related to renal lithiasis, pyelonephritis or lumbar and abdominal pain, the others were asymptomatic during the course of disease. The normal urothelial epithelium is capable of metaplasia into squamous, columnar, or cuboidal form ${ }^{7}$. Gangane $\mathrm{N}$ and colleagues thought that mucinous cystadenoma, like its malignant counterpart, arises in foci of intestinal metaplasia, because histological transition from urothelial epithelium to metaplastic mucinous areas in the same lesion was observed ${ }^{8}$. In the present case, we were able to demonstrate the transition from urothelium to mucinous columnar epithelium by histopathological examination. A few cases of mucinous cystadenoma arising from the renal pelvis had progressed to mucinous adenocarcinomas ${ }^{9,10}$. Gangane $\mathrm{N}$ et al reported one case of mucinous cystadenoma arising from renal pelvis, with lining pseudostratified epithelium with mucinous metaplasia and were thrown into delicate papillary folds in some places. This epithelium was reminiscent of the lining epithelium of mucinous cystadenoma of the ovary, pancreas and biliary tree; however, renal mucinous cystadenomas lack ovarian like stroma characteristic of the ovarian, pancreatic, and biliary mucinous cystic tumors ${ }^{8}$. Ross DG et al suggested that the same criteria used for evaluating mucinous ovarian tumors might also be appropriate for mucinous tumors of the kidney $^{11}$. Based on the clinical and morphological findings, we consider this case as a mucinous cystadenoma arising from the renal pelvis with calcification. Long-standing chronic inflammation and renal calculi have been invoked as possible etiologic factors in mucinous adenocarcinoma of the pelvis ${ }^{12}$. 
It seems that the same hypothesis holds true for mucinous adenomas as well. In fact, renal calculi were reported in 10 cases of primary renal mucinous cystadenoma in the previous reports ${ }^{1}$. However, this theory is not likely to be valid for the present case, because there is no prior history of urolithiasis or pyelonephritis. Arakawa et al believe in the theory that relies on glandular metaplasia in the kidney associated with urolithiasis and pyelonephritis ${ }^{13}$. By contrast, Liwinicz and associates have postulated that the formation of stones is secondary to the mucin secretion by the tumor. They have proposed that the mucins fused with sodium, calcium, and magnesium cations leading to the formation of stones.

Thus calculi may be the result, and not the cause of the neoplasm ${ }^{14}$. Interestingly, three cases of renal mucinous cystadenomas were discovered in horseshoe kidneys and all were of parenchymal origin. Such anomalous kidneys may have a tendency to have mucinous tumors of parenchymal origin. Some authors suggested that it was possible for such tumors to originate from a sequestered segment of renal pelvic epithelium in renal parenchyma, especially in an anomalous kidney ${ }^{2,5,11}$. It's very difficult to distinguish a mucinous cystadenoma from a simple renal parenchymal cyst based on routine imaging studies, including ultrasonography, CT, and MRI, and mucinous cystadenoma of the kidney may be more frequent than is diagnosed. It seems that treatment of renal mucinous cystadenoma should be individualized according to the radiological findings, since there are no definite diagnostic radiological criteria and treatment may not be necessary in some cases. Radiological studies may not be able to identify malignant mucinous cystic neoplasms of kidney, so complex renal cystic lesions should be carefully followed for early detection of malignancy ${ }^{15,16}$. If there is suspicion of malignancy, total or partial nephrectomy should be performed accordingly, the final diagnosis was made only after tumor excision. In summary, it suggests that mucinous cystadenoma, like its malignant counterpart, may arise in foci of metaplastic mucinous areas. The significance of this current special case lies in its radiologic features, which resembles hydronephrosis with non functioning kidney. It is difficult to suspect the diagnosis before surgery. Further follow-up is needed to determine the prognosis for such extremely rare renal neoplasm.

\section{References :}

1. Xiang $\mathrm{H}$, Zhang $\mathrm{X}, \mathrm{Ba} \mathrm{X}, \mathrm{Wu} \mathrm{W}$. Mucinous cystadenoma with calcification arising from renal pelvis radiologically resembled renal calculus with hydronephrosis: report of a rare case and review of the literature. Int J Clin Exp Pathol. 2017; 10(8):875660.

2. Chablé-Montero F, Mendoza-Ramírez S, Lavenant-Borja MI, González-Romo MA, SotoAbraham V, Henson DE, et al. Mucinous cystadenoma of the pelvicaliceal system: a report of 3 examples and an analysis of 17 previously published cases. Ann Diagn Pathol. 2013; 17:239-44.

3. Mitome T, Yao M, Udaka N, Fusayasu S, Izumi K, Osaka K, et al. Mucinous cystadenoma of a horseshoe kidney: a case report and literature review. Can Urol Assoc J. 2015; 9:E30-32.

4. Arcadi JA. Mucus-producing cystadenocarcinoma of the renal pelvis and ureter; fourth reported case. AMA Arch Pathol. 1956; 61:264-68.

5. Moch H, Humphrey PA, Ulbright TM, Reuter VE. World Health Organization. Classification of tumors of the urinary system and Male Genital Organs: glandular neoplasms. Lyon: IARC Press; 2016. pp. 111-112.

6. Akan H, Dalva I, Yildiz O, Kutluay L, Gündoğdu S, Güngen Y. Mucinous cystadenoma mimicking simple renal parenchymal cyst in a horseshoe kidney. Int J Urol. 2005; 12:493-96.

7. Ross DG, D'Amato NA. Papillary mucinous cystadenoma of probable renal pelvic origin in a horse shoe kidney. Arch Pathol Lab Med. 1985; 109:954-55.

8. Mostofi FK. Potentialities of bladder epithelium. J Urol. 1954; 71:705-14.

9. Gangane N, Anshu, Shende N, Sharma SM. Mucinous cystadenoma arising from renal pelvis: a report of 2 cases. Urol J. 2008; 5:197-99.

10. Toyoda H, Mabuchi T, Fukuda K. Mucinous cystadenoma with malignant transformation arising in the renal pelvis. Pathol Int. $1997 ; 47: 174-78$

11. Mardi K, Sharma J, Mahajan P. Mucinous cystadenoma of the renal pelvis with malignant transformation: a case report. Indian $\mathrm{J}$ Pathol Microbiol. 2006; 49:595-96.

12. Kobayashi S, Ohmori M, Akaeda T, Ohmori H, Miyaji Y. Primary adenocarcinoma of the renal pelvis. Report of two cases and brief review of literature. Acta Pathol Jpn.1983; 33:589-597.

13. Arakawa M, Jimi A, Ootomi M, Ooyabu Y, Samejima H. A mucin-producing cystadenoma, borderline malignancy, of the renal pelvis and ureter: a case report. Gan No Rinsho. 1989; 35:499-504.

14. Liwnicz BH, Lepow H, Schutte H, Fernandez R, Caberwal D. Mucinous adenocarcinoma of the renal pelvis: discussion of possible pathogenesis. J Urol. 1975; 114:306-10.

15. Kaur G, Naik VR, Rahman MN. Mucinous adenocarcinoma of the renal pelvis associated with lithiasis and chronic gout. Singapore Med J. 2004; 45:125-26. 